\title{
Resting Metabolic Rate and Anthropometric Measurements in Male Sleep Apnea Patients
}

\author{
Kagan Ucok ${ }^{1}$, Abdullah Aycicek ${ }^{2}$, Murat Sezer ${ }^{3}$, Fatma Fidan ${ }^{3}$, Lutfi Akgun ${ }^{1}$, \\ Muzaffer Akkaya $^{1}$ and Mehmet Unlu ${ }^{3}$
}

\begin{abstract}
Objective Obstructive Sleep Apnea Syndrome (OSAS) is a common sleep-related breathing disorder. Associations among Apnea-Hypopnea Index (AHI), Resting Metabolic Rate (RMR), body habitus differences, and otorhinolaryngologic abnormality may clarify the characteristics of patients with OSAS. In order to test this hypothesis, we aimed to compare the RMR, Modified Mallampati Scores (MMS), anthropometric measurements and body composition of male OSAS patients with simple snorers and to investigate the association among these parameters.

Methods MMS were calculated, overnight polysomnography was performed, body mass index, neck, shoulder, chest, waist, hip and abdomen circumferences, body fat amount and ratio, lean body weight, body water amount and RMR were measured for all of the patients.

Patients Ninety-eight male patients with suspected OSAS were included in the study.

Results Fifty-one patients were diagnosed as OSAS and 47 patients were diagnosed as simple snorers. RMR, total body water, neck, shoulder and chest circumferences were significantly higher in OSAS patients than the simple snorers. The majority of the simple snorers was seen to have a MMS of stage 2 whereas OSAS patients had MMS of stages 3 and 4. AHI was significantly correlated with neck, shoulder, chest circumferences, total body water amount, MMS and RMR. Chest girth was found as the most important single predictor of sleep apnea in multivariate analysis.

Conclusion We suggest that the increased RMR and chest circumference might have occured due to the condition resulting from the elevated AHI in patients with OSAS. Mallampati score should be routinely evaluated in OSAS patients.
\end{abstract}

Key words: obstructive sleep apnea, resting metabolic rate, anthropometry, body composition

(Intern Med 50: 833-838, 2011)

(DOI: 10.2169/internalmedicine.50.4779)

\section{Introduction}

Obstructive Sleep Apnea Syndrome (OSAS) is a common sleep disorder, with a prevalance of $3-4 \%$ in adult males $(1,2)$. Advanced age, anatomic variations, alcohol consumption, gender and obesity are important factors in the development of OSAS (3). It becomes more frequent with increasing age and Body Mass Index (BMI). The prevalence is 2-9 times higher in males than females $(2,4)$. The syndrome is charactarized by repetitive upper airway collapse during sleep. A pharyngeal airway which is narrow or has a tendency to collapse constitutes the pathogenetic basis of OSAS (2). The symptoms, which include excessive daytime sleepiness, nonrefreshing sleep, daytime fatigue, and decreased concentration, may cause marked impairment in social or occupational functioning. In addition to these symptoms that negatively affect quality of life, OSAS causes severe morbidity associated with cardiovascular and cerebrovascular systems (5). Polysomnography (PSG) is routinely indicated for the diagnosis of sleep-related breathing disorders including OSAS.

${ }^{1}$ Department of Physiology, Afyon Kocatepe University Faculty of Medicine, Turkey, ${ }^{2}$ Department of Otorhinolaryngology, Afyon Kocatepe University Faculty of Medicine, Turkey and ${ }^{3}$ Department of Pulmonary Medicine, Afyon Kocatepe University Faculty of Medicine, Turkey Received for publication November 2, 2010; Accepted for publication December 8, 2010

Correspondence to Dr. Kagan Ucok, kaganucok@ hotmail.com 
Obesity is recognized as a contributing factor to Sleep Disordered Breathing (SDB). There are numerous measures of obesity such as BMI and waist circumference. BMI is the most commonly used metric of obesity. However, it is not clear whether it is the best measure of obesity as it pertains to SDB. It is therefore asked how the different metrics of obesity, specifically BMI, neck circumference, waist circumference, and waist-hip ratio correlate with metrics of SDB, specifically with AHI, and which is most likely to predict and correlate with SDB diagnosis and treatment (2).

In addition to increased body weight, fat distribution plays a major role in the development of OSAS. Visceral obesity has been recognized to be associated more often with OSAS than other forms of obesity $(6,7)$. Therefore, BMI and anthropomorphic measurements such as neck circumference, waist circumference, and skin fold thickness could be used to predict the presence of OSAS and its severity. Along with BMI and neck circumference, adipose tissue quantification of the abdominal and neck regions with magnetic resonance imaging or computed tomography scanning was found also to be an important factor for predicting the severity of OSAS or AHI $(7,8)$. Bioelectrical impedance analysis (BIA) is one of the easiest methods to determine body composition. It measures the amount of fat tissue and fat free tissue by the difference of their resistance to electrical current.

Resting metabolic rate (RMR) is the component of energy expenditure that explains the largest proportion (70-80\%) of an individual's total daily energy expenditure (9). RMR is assessed by indirect calorimetry by measurements of oxygen consumption and carbon dioxide production. There are several potential mechanisms whereby OSAS might alter energy expenditure. Resting energy expenditure is reduced during sleep and could be similarly affected by daytime hypersomnolence $(10,11)$.

In this study, we aimed to compare the RMR, Modified Mallampati Scores (MMS), anthropometric measurements and body composition of male OSAS patients with simple snorers and to investigate the association among these parameters.

\section{Materials and Methods}

\section{Subjects}

Ninety-eight male patients, who were referred to Afyon Kocatepe University School of Medicine, Departments of Otorhinolaryngology and Pulmonary Medicine with suspected OSAS were included in the study. The study protocol was approved by the Ethical Committee of Afyon Kocatepe University, and all patients participated voluntarily with written informed consent. Patients who had craniofacial abnormality, retrognathism, cardiovascular diseases-particularly those who used beta blockers-metabolic disorders such as hypothyroidism and diabetes mellitus were excluded from the study.

\section{Otorhinolaryngologic examination}

All patients underwent otorhinolaryngologic examination. MMS of Friedman was evaluated as follows (12); the size of the tongue was assessed with regard to that of the soft palate while the tongue rests in the floor of the mouth. Stage 1: complete visualization of the uvula, tonsils and palatal arches. Stage 2: complete visualization of the uvula while the tonsils and arches are partially invisible. Stage 3: only the soft and hard palate are visible, and the uvula is hidden. Stage 4: only the hard palate is visible.

\section{Polysomnography}

Overnight PSG was arranged for all 98 patients. An overnight sleep study was performed in all patients using 18channel polysomnography (SleepScreen Pro ${ }^{\circledR}$, Viasys Healthcare, Hoechberg, Germany), which included a standard montage of electroencephalogram (EEG), electrooculogram and electromyogram signals together with pulse oximetry, respiratory impedance and nasal airflow detected by oronasal thermistors. All sleep studies were manually analyzed using computer software by the same physician who was not informed about the patient's other clinical and ultrasonographic data. Sleep stages were scored according to the standard criteria of Rechtschaffen and Kales (13). Apneas were defined as complete cessation of airflow for at least 10 sec. Hypopnea was defined as $\geq 50 \%$ reduction in airflow accompanied by $>3 \%$ desaturation in the preceding $30 \mathrm{sec}$ and a reduction in chest wall movements. EEG arousals were not required to make the diagnosis of a respiratory event. Data were expressed as the AHI based on the number of apneas and hypopneas per hour slept, with an AHI $\geq 10$ events/ $h$ indicating a positive OSAS diagnosis (14).

\section{Anthropometric measurements}

Anthropomorphic measurements including BMI, neck, shoulder, chest, waist, hip and abdomen circumferences were measured for all of the patients. BMI was calculated as body weight divided by the square of height $\left(\mathrm{kg} / \mathrm{m}^{2}\right)$.

Circumference measurements were carried out at anatomical position with a $7 \mathrm{~mm}$ wide tape measure. The tape measure was held parallel to the ground and completely surrounded the part of the body, but it did not compress the subcutaneous fat tissue. Duplicate measurements were taken at each site and retest was done if duplicate measurements were not within $7 \mathrm{~mm}$ (15). The standardized sites were used for circumference measurements as follows (16); neck circumference was measured horizontally at just inferior the Adam's apple. Shoulder circumference was measured over maximum bulges of the deltoid muscles (just below the acromion) at the end of normal expiration. Chest circumference was measured horizontally at the level of fourth costosternal joints at the end of expirium. Waist circumference was measured at level of the narrowest part of torso at the end of expirium. Hip circumference was determined as the maximum value over the buttocks. Abdomen circumference 
Table 1. Patient Characteristics

\begin{tabular}{lccc}
\hline & $\begin{array}{c}\text { Simple Snorer } \\
(\mathrm{n}=47)\end{array}$ & $\begin{array}{c}\text { OSAS Patients } \\
(\mathrm{n}=51)\end{array}$ & p value* \\
\hline Age & $49.7 \pm 10$ & $48.0 \pm 8$ & $\mathrm{NS}$ \\
$\mathrm{BMI}\left(\mathrm{kg} / \mathrm{m}^{2}\right)$ & $32.3 \pm 6.1$ & $32.5 \pm 5.5$ & $\mathrm{NS}$ \\
$\mathrm{AHI}$ & $3.5 \pm 2.3$ & $44.8 \pm 26.8$ & 0.000 \\
Neck Circumference $(\mathrm{cm})$ & $39.4 \pm 2.1$ & $42.1 \pm 5.8$ & 0.027 \\
Shoulder Circumference $(\mathrm{cm})$ & $109.8 \pm 5.6$ & $114.2 \pm 8.7$ & 0.001 \\
Chest Circumference $(\mathrm{cm})$ & $98.1 \pm 4.5$ & $106.3 \pm 9.1$ & 0.000 \\
Abdomen Circumference $(\mathrm{cm})$ & $105.9 \pm 8.5$ & $109.1 \pm 13.4$ & $\mathrm{NS}$ \\
Waist Circumference $(\mathrm{cm})$ & $99.9 \pm 8.7$ & $103.9 \pm 11.3$ & $\mathrm{NS}$ \\
Hip Circumference $(\mathrm{cm})$ & $108.7 \pm 6.5$ & $109.1 \pm 7.9$ & $\mathrm{NS}$ \\
Waist/Hip Ratio & $0.99 \pm 0.4$ & $1.0 \pm 0.5$ & $\mathrm{NS}$ \\
Body Fat (\%) & $26.8 \pm 5.3$ & $27.9 \pm 6.9$ & NS \\
Body Fat Amount $(\mathrm{kg})$ & $26.9 \pm 8.7$ & $27.2 \pm 11.2$ & $\mathrm{NS}$ \\
Lean Body Weight $(\mathrm{kg})$ & $66.2 \pm 6.8$ & $66.7 \pm 9.7$ & $\mathrm{NS}$ \\
Total Body Water Amount $(\mathrm{kg})$ & $45.1 \pm 4.6$ & $49.5 \pm 6.7$ & 0.001 \\
RMR (kcal/day) & $1332.8 \pm 285.2$ & $1676.4 \pm 600.0$ & 0.001 \\
\hline
\end{tabular}

*Independent samples t-test. Mean \pm SD are shown. BMI: body mass index, AHI:apnea-hypopnea index, RMR: resting metabolic rate, NS: Not Significant.

was measured horizontally at the level of greatest anterior protuberance of abdomen (usually at umbilicus) at the end of expirium.

\section{Determination of body composition}

Body composition of the patients was determined by BIA (Bodystat 1500, Bodystat Ltd., Douglas, Isle of Man, UK). Heavy physical exercise was restricted 24 hours before the test. Impedance was measured between the right wrist and right ankle using a tetrapolar electrode system. The subjects lay supine with arms separated from the body, and with legs not touching each other. Signal electrodes were positioned in the middle of the dorsal surface of the hand and feet proximal to the metacarpophalangeal and metatarsophalangeal joints. Detecting electrodes were more proximally positioned at the ankle and the wrist. An excitation current of $500 \mu \mathrm{a}$ at $50 \mathrm{kHz}$ was applied to the distal electrodes, and the voltage was detected by proximal electrodes. The data were analyzed using the manufacturer's software, and body fat percentage, total body fat, lean body mass, total body water amount were determined for each patient.

\section{Resting metabolic rate}

Resting metabolic rate was measured with an indirect calorimeter (Quark b ${ }^{2}$, Cosmed, Rome, Italy). The subjects were instructed to avoid food intake for 12 hours and not to perform exercise for 24 hours before the test. The tests were performed at the same hours $\left(08^{30}-10^{30}\right)$ of the day. After resting for 15 minutes, the measurements were applied to the subjects in a silent, lightless laboratory which was at room temperature. The device was calibrated prior to each test. The subjects were asked to put on a face mask, lay in supine position and not to move their arms or legs during the test. RMR was measured indirectly in 15 - 20 minutes with a metabolic card by the analysis of respired gases $\left(\mathrm{O}_{2}\right.$ and $\mathrm{CO}_{2}$ ).

\section{Statistical analysis}

Data were evaluated using Statistical Package for the Social Sciences (SPSS) ver.16.0 program. Chi-square test, independent samples t-test, Pearson and Spearman correlation tests were used. Logistic regression analysis was used for multivariate analysis. A p value of less than 0.05 was considered to be statistically significant.

\section{Results}

Of the total 98 patients, 51 had an AHI score $\geq 10$ and were diagnosed as OSAS. The remaining 47 had an AHI score $<10$ and were diagnosed as simple snorers. The characteristics of all 98 patients are shown in Table 1. There was no significant difference for age, BMI, abdomen circumference and waist/hip ratio between the groups. Resting metabolic rate was significantly higher in OSAS patients than in the simple snorers. Neck, shoulder and chest circumferences were significantly higher in OSAS patients than in the simple snorers. With regard to the results of BIA measurements, lean body weight, body fat amount and ratio were not significantly different in OSAS patients than in the simple snorers. Total body water amount was significantly higher in OSAS patients than in the simple snorers.

MMSs of the control group and OSAS patients are shown in Fig. 1. The majority of the simple snorers was seen to have a MMS of 2 whereas OSAS patients had MMS of 3 and 4.

There was a significant correlation between AHI and neck, shoulder, chest circumferences. AHI was also significantly correlated with total body water amount and RMR (Table 2). The relation between AHI and RMR is shown in Fig. 2. 


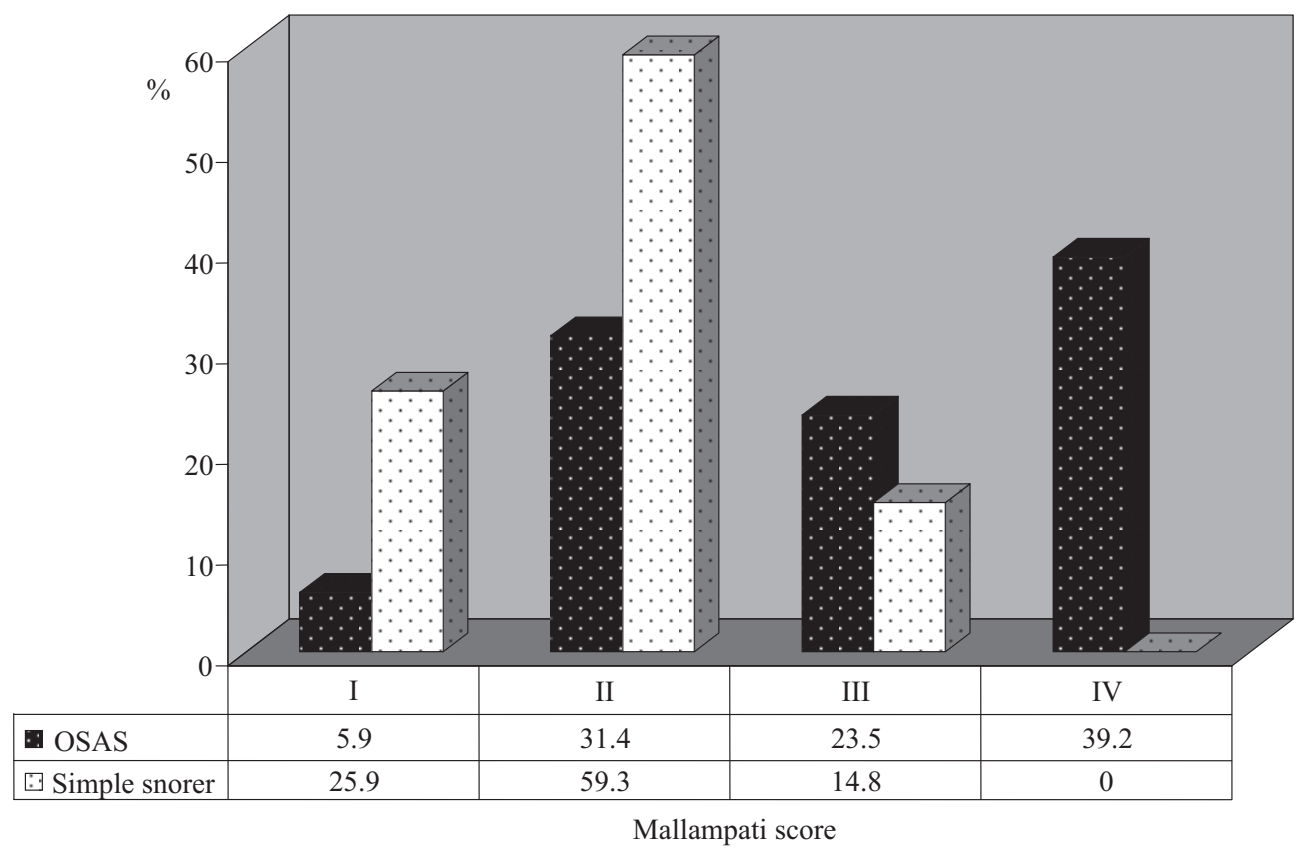

Figure 1. Modified Mallampati Scores of patients.

Table 2. Correlations between Anthropometric Measurements and AHI, MMS of OSAS Patients

\begin{tabular}{|c|c|c|c|c|}
\hline & \multicolumn{2}{|c|}{$\mathrm{AHI}$} & \multicolumn{2}{|c|}{ MMS } \\
\hline & rvalue* & p value* & rvalue** & $p$ value** \\
\hline Age & NS & NS & NS & NS \\
\hline $\mathrm{BMI}\left(\mathrm{kg} / \mathrm{m}^{2}\right)$ & NS & NS & NS & NS \\
\hline Neck Circumference $(\mathrm{cm})$ & 0.288 & 0.010 & 0.284 & 0.012 \\
\hline Shoulder Circumference $(\mathrm{cm})$ & 0.370 & 0.001 & 0.321 & 0.003 \\
\hline Chest Circumference (cm) & 0.430 & 0.000 & 0.303 & 0.006 \\
\hline Abdomen Circumference $(\mathrm{cm})$ & NS & NS & 0.287 & 0.011 \\
\hline Waist/Hip Ratio & NS & NS & NS & NS \\
\hline Body Fat (\%) & NS & NS & 0.296 & 0.008 \\
\hline Lean Body Weight (kg) & NS & NS & NS & NS \\
\hline Total Body Water Amount (kg) & 0.398 & 0.000 & 0.284 & 0.013 \\
\hline RMR (kcal/day) & 0.286 & 0.010 & 0.407 & 0.000 \\
\hline
\end{tabular}

*Pearson correlation test. ${ }^{*}$ Spearman correlation test. AHI: apnea-hypopnea index, MMS: modified Mallampati score, NS: Not Significant.

Multivariate analysis of anthropometric measurements (neck, shoulder, chest, abdomen, waist and hip circumferences, waist/hip ratio, BMI) revealed that chest circumference was the most important single predictor of OSAS (OR: 1.9, CI: 1.2-2.8, p: 0.003).

In addition, RMR was correlated with only shoulder circumference $(\mathrm{r}=0.294, \mathrm{p}=0.010)$, chest circumference $(\mathrm{r}=$ $0.431, \mathrm{p}=0.000)$, total body water amount $(\mathrm{r}=0.366, \mathrm{p}=$ 0.001 ) after controlling for age and BMI.

MMS was significantly correlated with AHI ( $r=0.584$, $p=$ 0.000). MMS was also significantly correlated with neck, shoulder, chest and abdomen circumferences, body fat \%, total body water amount and RMR (Table 2).

\section{Discussion}

Studies in rats and normal human subjects show that experimental sleep fragmentation is associated with increased energy expenditure $(17,18)$. Repetitive apneas with associated arousals from sleep and the increased work of breathing in patients with OSAS could similarly increase energy expenditure (19). Ryan et al found higher resting energy expenditure in OSAS patients than the controls (11). Stenlof et al studied five adults with OSAS and reported that sleeping energy expenditure and 24-h energy expenditure in a whole body calorimeter were significantly increased relative to controls, and declined after treatment (20). Marcus et al also demonstrated substantial declines in sleeping energy expenditure in 14 children with OSAS after adenotonsillec- 


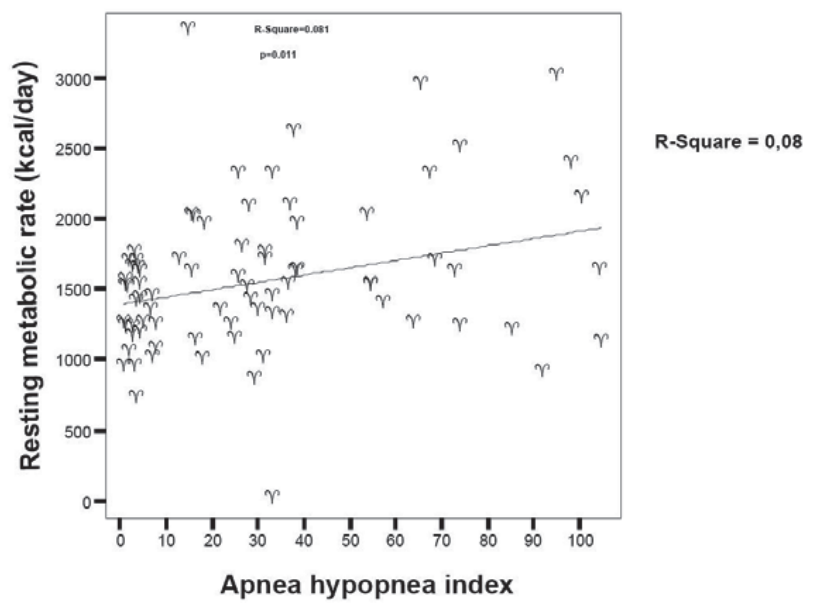

Figure 2. The relation between AHI and RMR.

tomy (19). Feng et al found that corticotropin-releasing hormone and cortisol levels in the OSAS group were significantly higher than those of the control group (21). Sleep loss can affect the stress response and may accelerate the development of metabolic and cognitive consequences of glucocorticoid excess (22). Sympathetic over-activity at both acute and diurnal (non-apneic) conditions were demonstrated with urinary and plasma catecholamines, and intra-neuronal recordings of muscle sympathetic nerve activity in patients with OSAS (23). Similar to the above-mentioned studies, we found that RMR was significantly higher in OSAS patients than in simple snorers (Table 1). Also, it was correlated with AHI but R-square was low (Table 2, Fig. 1). RMR might have been affected by the other factors together with AHI. We suggest that elevated RMR might be due to increased sympathetic activity as well as cortisol levels in OSAS patients.

Neck circumference is one of the well known and commonly used anthropometric parameters in assessing patients with OSAS $(24,25)$. In this study, neck, shoulder, chest circumferences were significantly higher in OSAS patients than in simple snorers (Table 1). In addition, they were correlated with AHI (Table 2). These might indicate possible uppertrunk obesity in OSAS patients. In a previous published study, we investigated aerobic capacity in OSAS patients, and we reported that upper-body subcutaneous fat (except for subcutaneous abdominal fat) was correlated with aerobic capacity and it might be suitable for determining the physical fitness of patients with OSAS (26). Similarly, in the current study the RMR was correlated with upper-trunk anthropometry (shoulder, chest circumferences) and body water amount. We think that the increases in neck, shoulder, chest circumferences and body water amount might be due to hormonal changes in OSAS, such as an increase in cortisol levels $(21,22,27)$. It is a well-known fact that elevated cortisol levels can cause an increase in neck and upper-trunk fat accumulation, thereby it may alter anthropometric measurements of this region. In another previously published study, we found that the antero-posterior diameter of the thorax was significantly larger in OSAS patients than those in simple snorers $(28,29)$. Interestingly, in the current study, chest circumference was found to be the single, most important predictor of sleep apnea by the multivariate analysis of anthropometric parameters. Some factors such as ethnic and body composition differences may be factors leading to this result. In addition, the increase of chest circumference in patients with OSAS might also be considered to be due to an increased respiratory effort of these patients during sleep as seen in a report in which the antero-posterior diameter of thorax was found to be larger in simple snorers (29). Although Park and Shin reported that the chest circumference of snorers was larger than that of non-snorers, to our knowledge, chest circumference has not been studied for OSAS patients before (30). We suggest that in evaluating patients with OSAS, upper-trunk anthropometry (especially chest circumference) should be taken into account.

Patients with severe SDB are often obese (31). Obesity, which is the most common predisposing factor increases the risk of OSAS development by approximately ten fold, from $2-4 \%$ to $20-40 \%$ in those with a BMI greater than 30 , and weight reduction may lead to a marked decline in sleep apnea severity (32). BMI has been the most widely used parameter to describe obesity. It is known that there is a strong correlation between the degree of sleep-related breathing disorders and anthropomorphic measurements of obesity. We found that BMI, waist/hip ratio, abdomen circumference, body fat percentage, body fat amount and lean body weight were not significantly different between OSAS patients and simple snorers (Table 1). In addition, BMI, waist/hip ratio, abdomen circumference, body fat percentage and lean body weight values were found to be not significantly correlated with AHI in all patients (Table 2). These findings set us thinking that, variations in body composition and anthropometry can not be the only explanation as to why all obese individuals do not develop OSAS.

The Mallampati score is generally used to evaluate patients for the possibility of difficult intubation during anesthesia. Hiremath et al studied the relationship between difficult endotracheal intubation and OSA and showed that patients with higher Mallampati grades had higher apnea/hypopnea scores (33). Friedman et al showed that MMS was highly predictive of estimating severity of OSA (12). Similarly, in our study, MMS was significantly higher in OSAS patients than in simple snorers (Fig. 1). Increased tongue size results in a higher Mallampati classification. Higher Mallampati scores correlate with a higher prevalence and severity of SDB. Therefore, increased fat deposition in the tongue increases tongue volume, thereby increasing the Mallampati score and contributing to SDB (34). This simple examination method was found to be correlated with the parameters that were also correlated with AHI (Table 2). MMS was also significantly correlated with AHI. We suggest that this simple examination method should be a routine procedure in evaluating OSAS patients. 


\section{Conclusions}

We found that RMR was significantly higher in OSAS patients than in simple snorers. Also RMR was associated with AHI and upper-trunk anthropometry in OSAS patients. Chest circumference was found to be the most important single predictor of sleep apnea and was suggested to be taken into account in assessing patients with OSAS. We suggest that the increased RMR and chest circumference might have occured due to the condition resulting from the elevated AHI in patients with OSAS. Mallampati score should be routinely evaluated in OSAS patients. Further studies are needed to evaluate anthropometry together with hormonal status such as cortisol levels in patients with OSAS.

The authors state that they have no Conflict of Interest (COI).

\section{References}

1. Lavie P. Incidence of sleep apnea in a presumably healthy working population: A significant relationship with excessive daytime sleepiness. Sleep 6: 312-318, 1983.

2. Young T, Palta M, Dempsey J, Skatrud J, Weber S, Badr S. The occurence of sleep disordered breathing among middle-aged adults. N Engl J Med 328: 1230-1235, 1993.

3. Andrews JG, Oei TP. The roles of depression and anxiety in the understanding and treatment of obstructive sleep apnea syndrome. Clin Psychol Rev 24: 1031-1049, 2004.

4. Block AJ, Boysen PG, Wynne JW, Hunt LA. Sleep apnea, hypopnea, and oxygen desaturation in normal subjects. A strong male predominance. N Engl J Med 300: 513-517, 1979.

5. Ogretmenoglu O, Suslu AE, Yucel OT, Onerci TM, Sahin A. Body fat composition: A predictive factor for obstructive sleep apnea. Laryngoscope 115: 1493-1498, 2005.

6. Grunstein R, Wilcox I, Yang TS, Gould Y, Hedner J. Snoring and sleep apnoea in men: association with central obesity and hypertension. Int J Obes 17: 533-540, 1993.

7. Shinohara E, Kihara S, Yamashita S, et al. Visceral fat accumulation as an important risk factor for obstructive sleep apnea syndrome in obese subjects. J Int Med 241: 11-18, 1997.

8. Schafer H, Pauleit D, Sudhop T, Gouni-Berthold I, Ewig S, Berthold HK. Body fat distribution, serum leptin, and cardiovascular risk factors in men with obstructive sleep apnea. Chest 122: 829-839, 2002.

9. Vogels N, Diepvens K, Westerterp-Plantenga MS. Predictors of long-term weight maintenance. Obes Res 13: 2162-2168, 2005.

10. Heller HC, Glotzbach SF. Thermoregulation and sleep. In: Environmental Physiologly II. Switzer A, Eberhart RC, Eds. University Park Press, Baltimore, 1977: 147-187.

11. Ryan CF, Love LL, Buckley PA. Energy expenditure in obstructive sleep apnea. Sleep 18: 180-187, 1995.

12. Friedman M, Tanyeri H, La Rosa M, et al. Clinical predictors of obstructive sleep apnea. Laryngoscope 109: 1901-1907, 1999.

13. Rechtschaffen A, Kales A. A Manual of Standardized Terminology, Techniques and Scoring System for Sleep Stages of Human Subjects. Washington DC. Public Health Services, NIH Publication no. 204, US Government Printing Office, 1968.
14. Lavie $P$, Herer P, Hoffstein V. Obstructive sleep apnoea syndrome as a risk factor for hypertension: population study. BMJ 320: 479482,2000

15. American College of Sports Medicine. ACSM's Health-Related Physical Fitness Assessment Manual. 2nd ed. Lippincott Williams \& Wilkins, USA, 2007: 48-49.

16. Heyward VH. Advanced Fitness Assessment and Exercise Prescription. 5th ed. Human Kinetics, USA, 2006: 337.

17. Bergmann BM, Everson CA, Kushida CA, et al. Sleep deprivation in the rat: V. Energy use and mediation. Sleep 12: 31-41, 1989.

18. Bonnet MH, Berry RB, Arand DL. Metabolism during normal, fragmented, and recovery sleep. J Appl Physiol 71: 1112-1118, 1991.

19. Marcus CL, Carroll JL, Koerner CB, Hamer A, Lutz J, Loughlin GM. Determinants of growth in children with the obstructive sleep apnea syndrome. J Pediatr 125: 556-562, 1994.

20. Stenölf K, Grustein R, Hedner J, Sjöström L. Energy expenditure in obstructive sleep apnea: Effects of treatment with continuous positive airway pressure. Am J Physiol 271: E1036-E1043, 1996.

21. Feng XW, Kang J, Wang ZF, Wang QY, Yu RJ. Regulation of hypothalamus-pituitary-adrenal axis and growth hormone axis in obstructive sleep apnea-hypopnea syndrome patients. Zhonghua Jie He He Hu Xi Za Zhi 29: 230-232, 2006.

22. Leproult R, Copinschi G, Buxton O, Van Cauter E. Sleep loss results in an elevation of cortisol levels the next morning. Sleep 20: 865-870, 1997.

23. Fletcher EC. Sympathetic over activity in the etiology of hypertension of obstructive sleep apnea. Sleep 26: 15-19, 2003.

24. Katz I, Stradling J, Slutsky AS, Zamel N, Hoffstein V. Do patients with obstructive sleep apnea have thick necks? Can Am Rev Respir Dis 141: 1228-1231, 1990.

25. Dixon JB, Schacter LM, O'Brien PE. Predicting sleep apnea and excessive day sleepiness in the severely obese. Chest 123: 11341141, 2003.

26. Ucok K, Aycicek A, Sezer M, et al. Aerobic and anaerobic exercise capacities in obstructive sleep apnea and associations with subcutaneous fat distributions. Lung 187: 29-36, 2009.

27. Bratel T, Wennlund A, Carlstrom K. Pituitary reactivity, androgens and catecholamins in obstructive sleep apnoea. Effects of continuous positive airway pressure treatment (CPAP). Respir Med 93: 17, 1999.

28. Ucok K, Aycicek A, Mollaoglu H, et al. Respiratory variables during wakefulness in patients with obstructive sleep apnea and associations with anthropometric measurements. J Physiol Sci 59 (Suppl 1): 266, 2009 (Meeting abstract).

29. Ucok K, Aycicek A, Mollaoglu H, et al. Respiratory variables during wakefulness in patients with obstructive sleep apnea and associations with anthropometric measurements. Eur J Gen Med 7: 250-258, 2010.

30. Park CG, Shin C. Prevalence and association of snoring, anthropometry and hypertension in Korea. Blood Pressure 14: 210-216, 2005.

31. Okada M, Takamizawa A, Tsushima K, Urushihata K, Fujimoto K, Kubo K. Relationship between sleep-disordered breathing and lifestyle-related illnesses in subjects who have undergone healthscreening. Intern Med 45: 891-896, 2006.

32. Kyzer S, Charuzi I. Obstructive sleep apnea in the obese. World J Surg 22: 998-1001, 1998.

33. Hiremath AS, Hillman DR, James AL, Noffsinger WJ, Platt PR, Singer SL. Relationship between difficult tracheal intubation and obstructive sleep apnea. Br J Anaesth 80: 606-611, 1998.

34. Nashi N, Kang S, Barkdull GC, Lucas J, Davidson TM. Lingual fat at autopsy. Laryngoscope 117: 1467-1473, 2007.

(C) 2011 The Japanese Society of Internal Medicine http://www.naika.or.jp/imindex.html 